Ann. Abeille, I966, 9 (3), 175-208

\title{
ÉTUDE BIOMÉTRIQUE DU POLLEN DES ESPÈCES FRANÇAISES DU GENRE ONOBRYCHIS
}

\author{
Michèle GENIER \\ Station de Recherches sur l'Abeille et les Insectes sociaux, \\ 91 - Bures-sur-Yvette
}

\section{SOMMAIRE}

L'étude statistique des grandeurs $\mathrm{P}$ (longueur de l'axe polaire) et $\mathrm{E}$ (diamètre du grain à l'équateur) se rapportant aux pollens des différentes variétés cultivées d'Onobrychis sativa, montre que les 6 variétés étudiées se classent en 2 groupes : variétés dites "longues " et variétés dites " courtes". La description des pollens des différentes espèces d'Onobrychis permet de constater que tous présentent les mêmes caractères morphologiques concernant le sporoderme et les apertures. Les mesures de $\mathrm{P}$ et $\mathrm{E}$ représentent donc les deux seuls critères permettant de différencier deux espèces.

\section{INTRODUCTION}

Le pollen des plantes du genre Onobrychis se rencontre de façon particulièrement fréquente dans tous les miels d'une grande partie de 1'Europe; par ailleurs, l'observation courante a montré que les abeilles sont très fortement attirées par ces plantes qui leur fournissent en abondance pollen et nectar.

Les miels purs de Sainfoin ne sont pas rares en dépit d'une nette régression des surfaces cultivées depuis environ un demi-siècle; on les trouve encore de façon assez régulière aussi bien dans les régions à sols calcaires du Bassin Parisien que dans certaines régions méridionales. Avec les Sainfoins, nous sommes donc en présence de plantes mellifères importantes qui représentent pour l'Abeille une source de nourriture abondante et régulière. Ils méritent de ce fait une étude particulière.

On peut distinguer dans le genre Onobrychis, tel qu'il se présente dans la flore française, deux groupes distincts : dans un premier groupe, peuvent être placées les sous-espèces et les variétés assez nombreuses d'Onobrychis sativa LAMk. ; le second groupe rassemblant toutes les autres espèces du genre apparaît beaucoup moins important pour l'Abeille.

Jusqu'ici, les travaux de mélisso-palynologie ont permis de mettre en évidence l'importance relative des Onobrychis au sein de la flore mellifère européenne, mais aucune précision n'a été apportée en ce qui concerne la présence ou l'absence des 
espèces autres qu'Onobrychis sativa LAMK., et aucun élément ne permet non plus de savoir si les variétés couramment cultivées sont toutes aussi attractives pour l'abeille. On sait qu'à l'intérieur d'une même espèce, des variétés morphologiquement très voisines peuvent présenter des caractères physiologiques très différents; il n'est donc pas exclu qu'au sein de l'espèce Onobrychis sativa I.AMK. il puisse exister des variétés présentant une sécrétion nectarifère quantitativement variable, ce qui entraînerait, de ce fait, une utilisation plus ou moins importante de ces variétés par 1'Abeille.

Notons encore que les spécialistes de l'analyse pollinique ont observé, sans toutefois approfondir le problème, des différences morphologiques assez nettes portant notamment sur les dimensions des pollens de Sainfoin rencontrés dans les différents miels au cours des analyses. Nous avons donc abordé cette étude en nous proposant de déterminer les caractéristiques morphologiques du plus grand nombre possible de pollens du genre Onobrychis. En raison des difficultés évidentes que nous allions rencontrer, nous avons pensé que le problème ne pouvait être abordé que par le biais d'une étude biométrique, seule susceptible de mettre en évidence de façon statistique des différences morphologiques, que nous supposions, dès le départ relativement minimes.

I,étude biométrique des grains de pollen du genre Onobrychis constitue la première étape de notre travail. Nous verrons ultérieurement qu'elle a abouti à la détermination précise d'un certain nombre de caractères, que nous avons ensuite utilisés pour tenter d'identifier les différents types morphologiques mis en évidence dans les populations polliniques rencontrées dans des miels provenant de régions géographiques bien déterminées.

Ainsi, nous pensons que notre travail permettra de compléter les données relatives aux pollens d'Onobrychis. En conséquence, il apportera aux spécialistes le moyen d'identifier dans les miels les différentse espèces d'Onobrychis que l'on peut y rencontrer, peut-être même certaines variétés d'Onobrychis sativa LAMK. Ceci pouvant permettre par la suite de mieux connaître le rôle respectif que jouent dans la nature les différents types et d'orienter éventuellement les recherches des généticiens qui se penchent actuellement sur le problème de sélection du Sainfoin.

\section{I. - GÉNÉRAIITÉS SUR IE GENRE “ ONOBRYCHIS » ET SES ESPÈCES PRINCIPALES}

\section{Considérations générales sur la culture du Saintoin. Distribution géographique. Variété.}

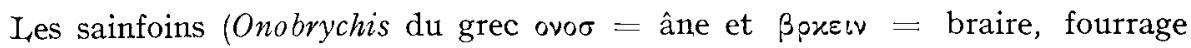
qui fait braire les ânes) constituent un gente comprenant une centaine d'espèces vivaces, de la famille des Papilionacées. Ce genre Onobrychis, rangé dans la tribu des Lotées, groupe des plantes herbacées ou parfois arbustives et épineuses, originaires du sud de l'Europe, de l'Afrique du Nord, de l'Asie occidentale.

Onobrychis sativa est une légumineuse fourragère précieuse dans les terrains 
pauvres, légers, calcaires; sa culture en effet, en tant que légumineuse, améliore les sols dans lesquels on la cultive.

Les sols qui conviennent le mieux à la culture du Sainfoin sont les limons cal caires asse $z$ secs et profonds, mais des sols beaucoup moins riches, à condition d'être profonds, lui conviennent également. La plante supporte la sécheresse et le froid, mais elle est assez sensible à 1'humidité persistante.

Il nous a semblé intéressant dans le cadre de ces généralités sur la culture du Sainfoin, d'essayer de dresser des cartes de distribution géographique de la plante. Ces cartes auront le mérite d'être plus "parlantes " qu'un long texte, elles nous permettront, par ailleurs, de relier ultérieurement distribution géographique de la plante et distribution mélissologique (fig. I, 2, 3, 4, 5, 6).

Les éléments nécessaires à la réalisation de ces cartes ont été empruntés aux Flores de G. Bonnier, de H. Coste, de P. Fournier, de M. Wilizomm et J. LAnge.

Les types cultivés de Sainfoin sont hétérogènes ; il est utile de distinguer trois grands types : le Sainfoin à une coupe dit "simple », celui à deux coupes dit "double » et enfin un type intermédiaire.

I. Le Sainfoin dit " simple » est aussi appelé Sainfoin commun : c'est une plante persistante, susceptible de donner trois ou quatre récoltes consécutives. Son développement est plus faible durant l'année du semis que celui du type double.

2. Le Sainfoin dit "double " est un type à développement plus considérable, fleurissant deux fois la même année, mais susceptible de ne fournir de récolte que pendant deux ans.

Le tableau I donne les caractères des variétés que nous avons pu étudier ici.

L'étude des cartes statistiques, empruntées à l'ouvrage de KLATzMANN (I955), nous montre qu'en France les superficies consacrées à la culture du Sainfoin ont diminué beaucoup de r 882 à r 949 .

En $\mathbf{I} 882$, la plante était cultivée sur une grande partie du territoire français, principalement dans les départements suivants : Calvados, Eure-et-Loire, Yonne, Aube, Marne, Loiret, Oise, Charente-Maritime, Charente, Vienne.

Or, en I949, seule la Vienne consacre encore une partie importante de son territoire à la culture du Sainfoin (plus de 24000 hectares).

Nous pouvons remarquer que, pendant les 60 dernières années, la culture du Sainfoin a périclité dans de nombreux départements de la moitié nord de la France, tandis qu’au contraire quelques départements, plus méridionaux, se sont davantage orientés vers la culture de cette plante, mais, de façon générale, la culture du Sainfoin semble être en régression en France, ceci vraisemblablement à cause de la nette diminution des élevages de chevaux et moutons. Pour ces animaux, en effet, le Sainfoin constitue un excellent fourrage.

\section{II. - MATÉRIEL, ET MÉTHODES}

\section{Méthodes générales de mélisso-palynologie}

Les méthodes utilisées pour la confection des préparations microscopiques de référence et pour celle des constituants figurés du miel sont celles qui ont été préconisées par la Commission internationale de Botanique apicole de l'Union internationale des Sciences biologiques (MAURIzIO et LOUVEAUX, 1963). 


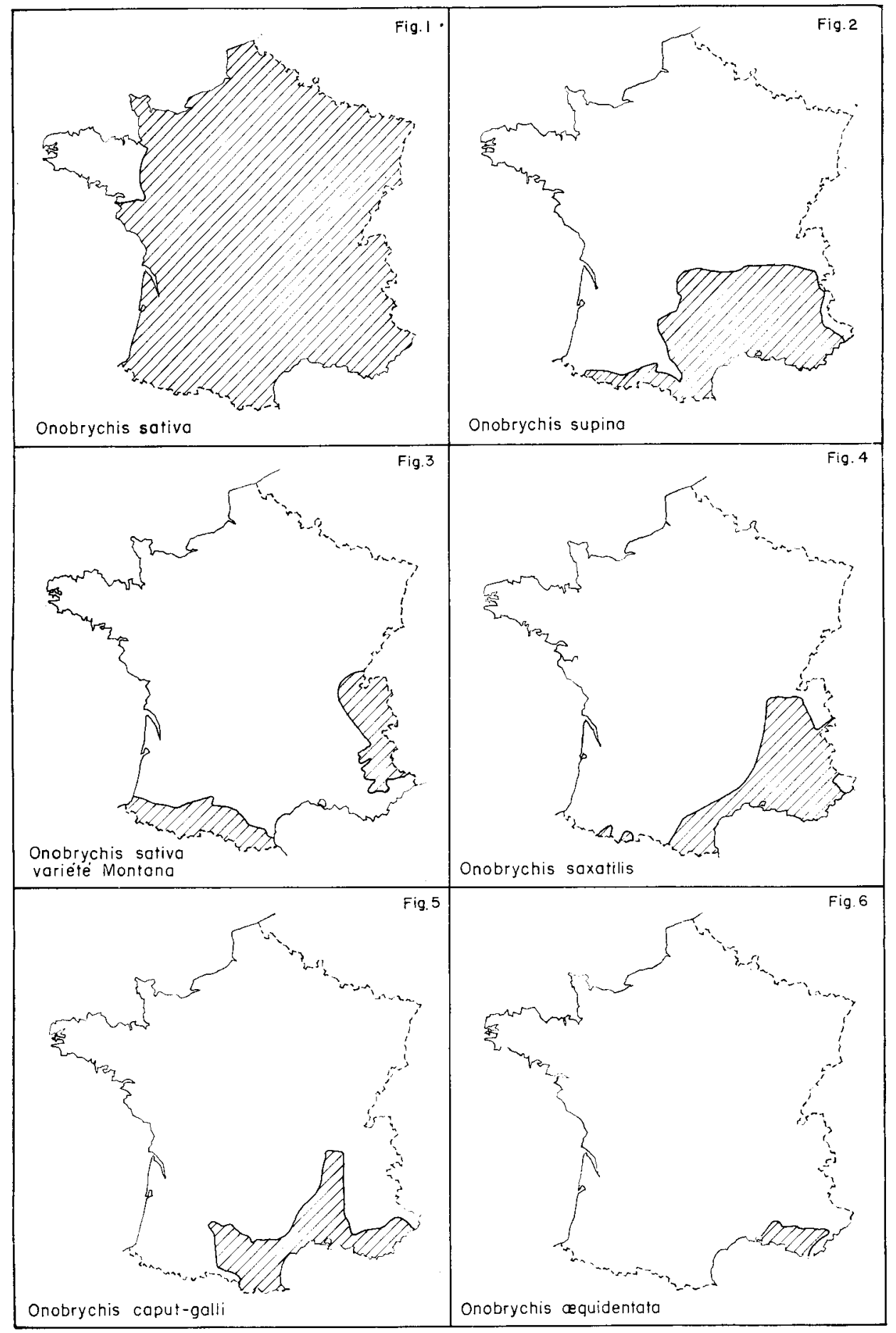

Fig. I à 6. - Répartition géographique des différentes espèces d'Onobrychis en France 


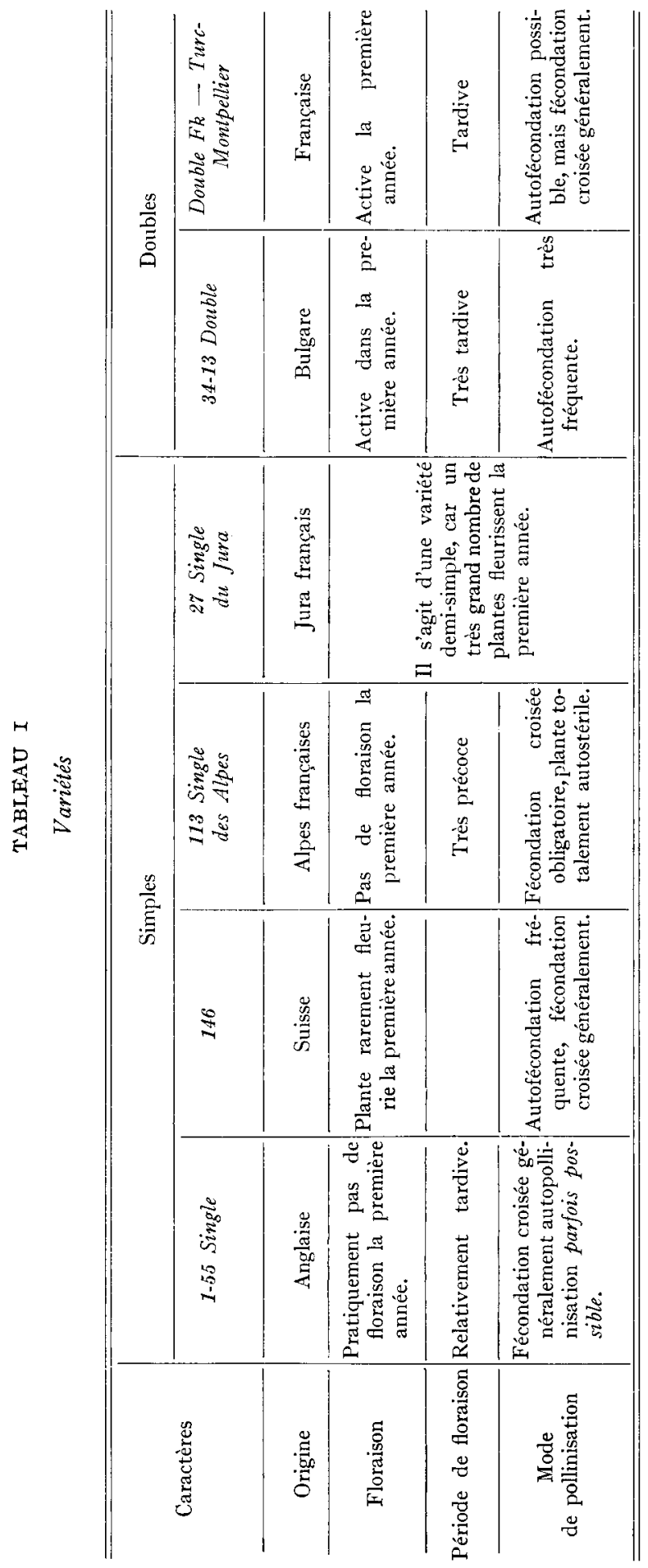




\section{Méthode de mensuration et calculs statistiques}

Les mesures effectuées portent dans chaque cas sur ıoo grains, $n=$ Io0.

Pour chaque grain d'une espèce ou d'une variété donnée, nous avons mesuré :

$\mathrm{P}=$ longueur de l'axe polaire.

$\mathrm{E}=$ diamètre du grain à l'équateur.

$\varepsilon=$ largeur du sillon germinal à l'équateur.

$t=$ distance séparant les extrémités de 2 sillons consécutifs.

Considérons, par exemple, le paramètre $P$.

La valeur moyenne de $P$, soit $\bar{P}$, a été établie pour chaque espèce en mesurant 100 grains, ce qui a permis, par ailleurs, de calculer l'espace de variation de cette grandeur. Puis nous avons essayé de voir si la différence trouvée concernant la même grandeur $\mathbf{P}$, lorsqu'on s'adresse à une autre espèce, est significative.

Nous avons enfin essayé d'établir une corrélation entre les variations de $\mathrm{P}$ et de $\mathrm{E}$ pour une même espèce.

\section{Calcul de la valeur moyenne de $P$, soit $\vec{P}$ à l'intérieur d'une espèce donnée.}

Les valeurs trouvées ont été rangées en classes successives croissant de $2 \mu$ en $2 \mu: x, x+2$, $x+4, \ldots$. ; nous avons appelé $p$ la valeur moyenne de la classe comptant un nombre variable $f$ de grains.

Sachant que $n$ - nombre total des grains mesurés - est toujours égal à Ioo, nous avons calculé la moyenne $\overline{\mathrm{P}}$ de la variable $\mathrm{P}$.

Calcul de l'espace de variation de la variable $P$.

Nous avons calculé sur nos données numériques la variance (v) et l'écart-type ( $\sigma$ ) ou erreur standard à la moyenne.

$$
\mathrm{S}_{m}=\frac{\sigma}{\sqrt{n-\mathrm{I}}}
$$

Dans ces conditions, 96 p. Ioo des valeurs moyennes de $\mathrm{P}$ se rapportant à $n$ grains de pollen de l'espèce considérée et tirés au hasard seront comprises entre $\overline{\mathrm{P}}-2,6 \mathrm{~S}_{m}$ et $\overline{\mathrm{P}}+2,6 \mathrm{~S}_{m}$.

Les valeurs $\overline{\mathrm{P}}+2,6 \mathrm{~S}_{m}$ constituent les limites de l'intervalle de confiance cherché où l'espace de variation correspond au coefficient de sécurité 96 p. Ioo.

Enfin, nous avons calculé par les méthodes habituelles le coefficient de corrélation reliant les paramètres $\mathrm{P}$ et $\mathrm{E}$.

Notons, avant d'aborder la description des grains, que dans le but d'attribuer aux résultats statistiques une précision suffisante nous nous sommes assurés que les conditions expérimentales de confection des préparations ne modifiaient pas les dimensions et les caractères observés, ainsi :

- le passage plus ou moins prolongé des lames sur la platine chauffante, le traitement plus ou moins poussé des grains par de plus ou moins grandes quantités d'éther ne modifient en rien la morphologie des pollens observés;

- de même que ne semble pas intervenir non plus dans ces considérations l'hydratation ou la non hydratation préalable des pollens et ceci, que l'on s'adresse à du pollen frais prélevé sur les anthères de plantes en fleurs ou à du pollen d'herbier.

Ainsi pour la variété cultivée d'Onobrychis sativa LAMK. 1-55 Single :

Les mesures effectuées sur du pollen non hydraté nous donnent les résultats suivants :

$$
\overline{\mathrm{P}}=33,24 \pm 0,33 \mu \quad \text { et } \quad \overline{\mathrm{E}}=23,28 \pm 0,28 \mu
$$

Les résultats des mesures effectuées sur du Pollen hydraté sont très voisines :

$$
\overline{\mathrm{P}}=33,64 \pm 0,39 \mu \quad \text { et } \quad \overline{\mathrm{E}}=23,23 \pm 0,3^{6} \mu
$$




\section{III. - RÉSULTATS}

\section{I. - IE POLLEN DES “ONOBRYChIS》}

Nous étudierons successivement les pollens de différentes espèces et variétés d'Onobrychis :

\section{A -- Onobrychis sativa LAMARK}

Préparations faites à Bures-sur-Yvette, en I964, à partir d'échantillon fourni par le Muséum d'Histoire naturelle de Paris, et provenant de l'Aisne.

Symétrie et forme.

Pollens isopolaires, tricolpés, nettement prolates, circulaires en vue polaire.

Dimensions.

Apertures.

$$
\begin{aligned}
& \overline{\mathrm{P}}=35,64 \pm 0,26 \mu \\
& \overline{\mathrm{E}}=2 \mathrm{I}, 32 \pm 0, \mathrm{I} 8 \mu
\end{aligned}
$$

Trois sillons, chaque sillon porte en son milieu une bande très étroite, sombre, presque continue d'ectexine, particulièrement visible en vue méridienne. Les bords des sillons sont réguliers et nets, les costae sont peu épaisses (entre I,5 et $2,3 \mu$ ), l'étalement de la costae est compris entre I,6 et 3, I $\mu$.

$$
\begin{aligned}
& t=3,4 \pm 0,8 \mu \\
& t=4,5 \pm 0,8 \mu
\end{aligned}
$$

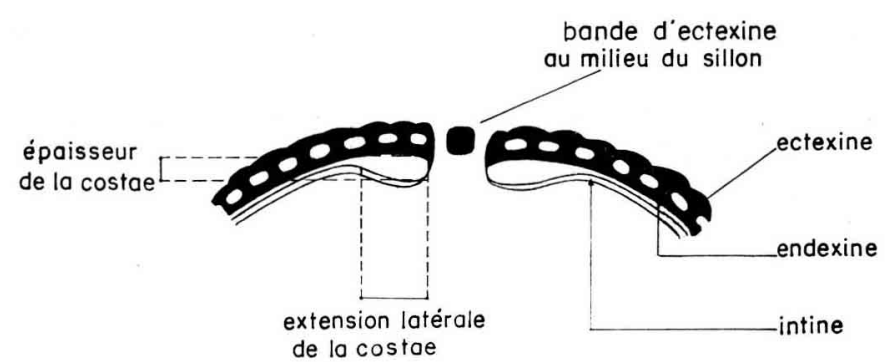

Fig. 7. - Coupe dans une aperture

Exine.

Réticulée, le réseau est régulier, les muri sont fins, les mailles du réseau ou lumina ont un diamètre inférieur à $x, 55 \mu$ et sont plus petites le long du sillon que dans l'intercolpium.

\section{Intine.}

Mince et claire.

L'ensemble exine + intine mesure environ $\mathrm{I}, 55 \mu$. 
Cytoplasme.

Clair et légèrement granuleux.

\section{Etude morphologique de la structure de l'exine.}

Il nous a semblé bon, étant donné le caractère complexe que revêt l'étude de la structure fine de l'exine sur pollen frais, de procéder à une fossilisation artificielle d'un certain nombre de grains de pollen selon la méthode de G. ERDTMAN (I954), fossilisation que nous avons fait suivre d'une chlorination de façon à obtenir des grains entièrement vidés de leur cytoplasme. Le pollen d'Onobrychis apparaît alors tecté-réticulé.

A la lumière de ces observations nous avons tenté de proposer une structure possible, bien sûr hypothétique, du sporoderme, le pouvoir séparateur du microscope optique ne permettant pas de détailler davantage cette structure. Il est, en effet, possible que les muri apparaissent pleins du fait de l'insuffisance du pouvoir séparateur du microscope optique et cette apparence peut résulter de l'accolement de columelles successives, l'espace séparant ces columelles étant trop petit pour être discernable.

Étude des différentes variétés cultivées d'Onobrychis sativa.

Onobrychis sativa constitue un groupe très homogène, les caractères morphologiques des grains de pollen des variétés cultivées en France sont identiques, seules varient les dimensions $P$ et $E_{1}$.

Les caractéristiques principales des différentes variétés étudiées ci-dessous ont été réunies dans le tableau $\mathrm{x}$.

\section{La variété I-55 Single.}

L'étude biométrique de cette variété sera traitée de façon détaillée à titre d'exemple.

Calcul de $\mathrm{P}$, selon la méthode expliquée précédemment.

$n=$ roo grains de pollen.

Différentes classes réunissant les valeurs de :

$$
\begin{aligned}
& \text { P p f } \\
& \frac{30,005+32,005}{2}=31,005 \times 12=392,37^{\circ} \\
& \frac{32,005+34,005}{2}=33,005 \times 23=759, \text { I I5 } \\
& \frac{34,005+36,005}{2}=35,005 \times 59=2065,295 \\
& \frac{36,005+38,005}{2}=37,005 \times 4=148,020 \\
& \text { Total }=3364,800
\end{aligned}
$$

la moyenne $\overline{\mathrm{P}}=\frac{3364,800}{\mathrm{I00}}=33,64 \mu$. 
Calcul de la variance $v$ :

$$
\begin{gathered}
v=\frac{12(33,648-31,005)^{2}+23(33,648-33,005)^{2}+}{59(33,648-35,005)^{2}+4(37,005-33,648)^{2}} \\
\text { I00 } \\
v=\frac{247, \mathrm{II} 2}{\mathrm{IOO}}=2,47 .
\end{gathered}
$$

Ce résultat permet de calculer $\sigma$ l'écart-type :

$$
=\mathrm{I}, 53
$$

ainsi l'erreur standard $S_{m}$

$$
\begin{gathered}
\mathrm{S} m=\frac{\mathrm{I}, 53}{\sqrt{n-\mathrm{I}}}=\frac{\mathrm{I,53}}{\sqrt{99}}=\frac{\mathrm{I}, 53}{9,94}=0, \mathrm{I} 53 \\
2,6 \mathrm{~S} m=0,398 .
\end{gathered}
$$

La valeur de $\overline{\mathrm{P}}$ est donc :

$$
33,64 \pm 0,39 \mu .
$$

Les mêmes calculs sont faits pour $E$.

$$
\begin{aligned}
& \text { Différentes classes } \quad e \quad f \\
& \frac{\mathrm{I7}, 005+\mathrm{I9,005}}{2}=\mathrm{I} 8,005 \times \mathrm{I}=\mathrm{I}= \\
& \frac{19,005+21,005}{2}=20,005 \times 2=40,010 \\
& \frac{2 \mathrm{I}, 005+23,005}{2}=22,005 \times 22=484, \mathrm{IIO} \\
& \frac{23,005+25,005}{2}=24,005 \times 73=175^{2,365} \\
& \frac{25,005+27,005}{2}=26,005 \times 0=1 \\
& \frac{27,005+29,005}{2}=28,005 \quad \times \quad \mathrm{I}=28,005 \\
& \frac{29,005+31,005}{2}=30,005 \times 0=1 \\
& \frac{3 \mathrm{I}, 005+33,005}{2}=32,005 \quad \times \quad I=32,005 \\
& \text { Total }=2322,495
\end{aligned}
$$


donc

$$
\mathrm{E}=\frac{2322,495}{100}=23,23 \mu
$$

$$
\begin{aligned}
\text { La variance } v & =\frac{223,530}{100}=2,24 \\
\text { L'écart type } \sigma & =\sqrt{2,24}=\mathrm{I}, 49
\end{aligned}
$$

$$
\begin{aligned}
\text { L'erreur standard } \mathrm{S} m & =\frac{\mathbf{I}, 49}{\sqrt{99}}=0, \mathrm{I} 4 \\
2,6 \mathrm{~S} m & =0,364
\end{aligned}
$$

$$
\mathrm{E}=23,23 \pm 0,36 \mu
$$

En groupant les valeurs par classes, nous pouvons Tréunir les résultats sous forme d'un tableau à double entrée, qui constitue un tableau de corrélation.

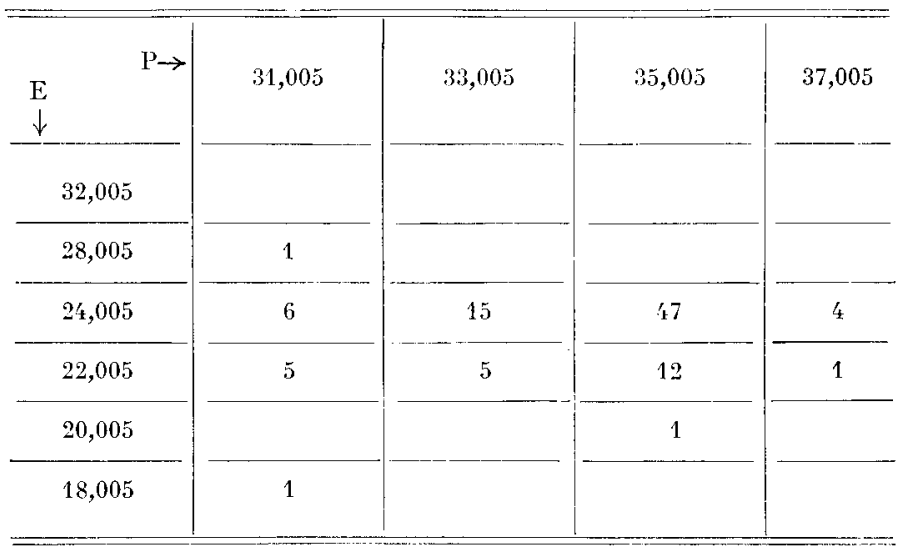

Calcul de la covariance p.

$$
p=\frac{\Sigma(e-\overline{\mathrm{E}})(p-\overline{\mathrm{P}})}{n}
$$

$$
\begin{aligned}
\Sigma= & (32-33,6)(28-23,2)+6(3 \mathrm{I}-33,6)(24-23,2)+\mathrm{I} 5(33-33,6)(24- \\
& 23,2)+47(35-33,6)(24-23,2)+4(37-33,6)(24-23,2)+5(3 \mathrm{I}- \\
& 33,6)(22-23,2)+5(33-33,6)(22-23,2)+\mathrm{I} 2(35-33,6)(22-23,2+ \\
& (37,33,6)(22,23,2)+\mathrm{I}(35-33,6)(20-23,2)+\mathrm{I}(3 \mathrm{I}-33,6)(\mathrm{I} 8-23,2) \\
= & -74,40+82,72 \\
\Sigma= & +8,32
\end{aligned}
$$

$$
p=\frac{8,32}{100}=+0,08
$$

le coefficient de corrélation $\quad r=\frac{p}{\sigma \mathrm{P} \overline{\sigma \mathrm{E}}}$

$$
r=\frac{0,08}{1,53 \times 1,49}=+0,03 \text {. }
$$


Le même plan sera suivi pour l'étude de chacune des variétés étudiées.

2. La variété $34-13$ double.

Dimensions :

$$
\begin{aligned}
& \overline{\mathrm{P}}=34,72 \pm 0,59 \mu \\
& \overline{\mathrm{E}}=23,57 \pm 0,27 \mu
\end{aligned}
$$

\begin{tabular}{|c|c|c|c|c|c|}
\hline $\begin{array}{l}\mathbf{E} \\
\downarrow \\
\end{array}$ & 33,005 & 35,005 & $: 37,005$ & 39,005 & 11,005 \\
\hline 27,005 & & & 22 & 3 & \\
\hline 25,005 & 1 & 17 & 8 & 9 & \\
\hline 23,005 & 1 & 16 & 13 & 10 & 1 \\
\hline 21,005 & 1 & 11 & t & 2 & 1 \\
\hline
\end{tabular}

Tableau de corrélation :

La covariance $p=+2,33$.

Le coefficient de corrélation $r=+0,9$.

3. La variété $\mathrm{x} 46$.

Dimensions :

$$
\begin{aligned}
& \overline{\mathrm{P}}=37,04 \pm 0,72 \mu \\
& \overline{\mathrm{E}}=22,25 \pm 0,36 \mu
\end{aligned}
$$

\begin{tabular}{|c|c|c|c|c|c|c|}
\hline $\begin{array}{ll} & \mathrm{P} \rightarrow \\
\mathrm{E} & \\
\downarrow & \\
\end{array}$ & 31,005 & 33,005 & 35,005 & 37,005 & 39,005 & 41,005 \\
\hline 27,005 & & 1 & & 1 & 1 & \\
\hline 25,005 & & & & 1 & 4 & \\
\hline 23,005 & 1 & 1 & 10 & 15 & 13 & 3 \\
\hline 21,005 & 1 & 2 & $1 \mathbf{1}^{\prime}$ & 19 & 9 & 3 \\
\hline
\end{tabular}

Tableau de corrélation :

La covariance $=p=+0,13$.

Le coefficient de corrélation $r=+0,03$. 
4. La variété Double Fk Turc-Montpellier.

Dimensions :

$$
\begin{aligned}
& \overline{\mathrm{P}}=36,26 \pm 0,65 \mu \\
& \overline{\mathrm{E}}=2 \mathrm{I}, 57 \pm 0,23 \mu
\end{aligned}
$$

\begin{tabular}{|c|c|c|c|c|c|c|}
\hline $\begin{array}{l}\mathrm{E} \\
\downarrow\end{array}$ & $\mathrm{P} \rightarrow$ & 33,005 & 35,005 & 37,005 & 39,005 & 41,005 \\
\hline 23,005 & & & 4 & 4 & 4 & 3 \\
\hline 21,005 & & & 18 & 24 & 24 & 14 \\
\hline 19,005 & & 1 & 2 & 1 & & \\
\hline
\end{tabular}

Tableau de corrélation :

La covariance $p=-0,2$.

Le coefficient de corrélation $r=-0,08$.

5. La variété II3 Single des Alpes.

Dimensions :

$$
\begin{aligned}
& \overline{\mathrm{P}}=36,06 \pm 0,36 \mu \\
& \overline{\mathrm{E}}=2 \mathrm{I}, 38 \pm 0,33 \mu
\end{aligned}
$$

\begin{tabular}{|c|c|c|c|c|c|c|}
\hline $\begin{array}{ll} & \mathrm{P} \rightarrow \\
\downarrow & \\
& \end{array}$ & 31,005 & 33,005 & 35,005 & 37,005 & 39,005 & 41,005 \\
\hline 25,005 & & & 1 & & 1 & \\
\hline 23,005 & & & 19 & t & 2 & \\
\hline 211,005 & & & 29 & 7 & 7 & 1 \\
\hline 19,005 & 2 & 4 & & 1 & 1 & \\
\hline
\end{tabular}

Tableau de corrélation :

Covariance $p=-0,12$.

Coefficent de corrélation $r=-0,06$.

6. La variété 27 Single du Jura.

Dimensions :

$$
\begin{aligned}
& \overline{\mathrm{P}}=34, \text { Io } \pm 0,69 \mu \\
& \overline{\mathrm{E}}=20,44 \pm 0,52 \mu
\end{aligned}
$$


Tableau de corrélation :

\begin{tabular}{|c|c|c|c|c|c|c|}
\hline $\mathrm{P} \rightarrow$ & 28,005 & 30,005 & 32,005 & 34,005 & 36,005 & 38,005 \\
\hline 24,005 & & & 1 & 5 & 2 & 2 \\
\hline 22,005 & & 3 & 5 & 7 & 10 & 6 \\
\hline 20,005 & & 6 & 4 & 9 & 8 & 7 \\
\hline 18,005 & 1 & 6 & 7 & 3 & 5 & 1 \\
\hline 16,005 & & 2 & & & & \\
\hline
\end{tabular}

La covariance $p=+1,7 /$.

Coefficient de corrélation $r=+0,3$.

Les tableaux 2 et 3 regroupent les résultats obtenus pour les différentes variétés étudiées : valeurs de $\mathrm{P}$ et de $\mathrm{E}$, corrélation entre ces deux paramètres.

TABLEAU 2

\begin{tabular}{|c|c|c|}
\hline Variétés & $\mathrm{P}$ & $\mathrm{E}$ \\
\hline $1-55$ Single.......... & $33,64 \pm 0,39$ & $23,23 \pm 0,36$ \\
\hline 34-13 Double ......... & $34,72 \pm 0,59$ & $23,57 \pm 0,27$ \\
\hline $146 \ldots \ldots \ldots \ldots \ldots \ldots$ & $37,04 \pm 0,72$ & $22,25=0,36$ \\
\hline Double Fk-Turc Montpellier & $36,26 \pm 0,65$ & $21,57 \pm 0,23$ \\
\hline 113 Single des Alpes.... & $36,06 \pm 0,36$ & $21,38 \pm 0,33$ \\
\hline 27 Single du Jura...... & $34,10 \pm 0,69$ & $20,44 \pm 0,52$ \\
\hline
\end{tabular}

TABLEAU 3

\begin{tabular}{|c|c|c|}
\hline Variétés & $p$ & $r$ \\
\hline 1-55 Single........... & $+0,08$ & $+0,03$ \\
\hline 34-13 Double .......... & $+2,33$ & $+0,9$ \\
\hline $146 \ldots \ldots \ldots \ldots \ldots$ & $+0,13$ & $+0,03$ \\
\hline Double Fk-Turc Monlpellier & $-0,2$ & $-0,08$ \\
\hline 113 Single des Alpes ..... & $-0,12$ & $-0,06$ \\
\hline 27 Single du Jura ...... & $+1,72$ & $+0,3$ \\
\hline
\end{tabular}


Résultats des calculs de corrélation.

De façon générale, aucune corrélation ne semble exister entre les variations de $\mathbf{P}$ et de $E$.

Deux exceptions à mentionner cependant :

En ce qui concerne la variété $34-13$ double, on constate qu'aux plus grandes valeurs de $\mathrm{P}$ correspondent les plus grandes valeurs de E. Le grain semble varieı en volume : il s'agit d'une corrélation positive. Par ailleurs, une très légère corrélation positive est à noter concernant le 27 Single du Jura.

Il est intéressant de constater qu'en formant le rapport $\mathrm{E}$ pour les différentes variétés étudiées, deux types peuvent être distingués (tabl. 4) :

\begin{tabular}{c|c} 
Tariétés cultivées \\
d'Onobrychis saliva
\end{tabular} \mid \begin{tabular}{c} 
Rapport $\frac{\mathrm{P}}{\mathrm{E}}$ \\
\hline $1-55$ Single
\end{tabular}

Io Rapport $\frac{\mathrm{P}}{\mathrm{E}} \# 1,65$. Tel est le cas des variétés :

146, Double Fk Turc-Montpellier, 113 Single des Alpes, 27 Single du Jura.

Ces variétés seront dites "longues".

$2^{\circ}$ Rapport $\frac{\mathrm{E}}{\mathrm{P}} \# \mathrm{I}, 50$. Telles sont les variétés :

1-55 Single et 34-13 double

variétés dites « courtes ».

Il est intéressant de voir maintenant si la diférence entre les valeurs moyennes de $P$ et de $E$ concernant 2 variétés est significative. 
Dans cet ordre d'idées, nous considérons, par exemple :

Les variétés 1-55 Single et 34-13 double, et selon la méthode indiquée précédemment, nous calculons l'erreur standard, $S_{d}$, de la différence des moyennes et le facteur, $t$, nous permettant de savoir rapidement si la différence des moyennes est significative ou non.

I. Etude concernant le paramètre $P$ :

$$
\begin{aligned}
& \text { I - } 55 \text { Single } \quad \overline{\mathrm{P}}_{1}-33,64 \quad v_{1}=2,470 \quad n_{1}=\text { I00 } \\
& 34 \text { - I3 double } \quad \overline{\mathrm{P}}_{2}=34,72 \quad v_{2}=5,436 \quad n_{2}=\text { I00 } \\
& \mathrm{S} d=\sqrt{\frac{2,470}{\mathrm{IOO}}+\frac{5,436}{\mathrm{IOO}}}=0,28 \\
& t_{1,2}=\frac{34,720-33,648}{0,28}=3,8
\end{aligned}
$$

$t_{1,2}$ est donc supérieur à 2,6 .

Il s'ensuit que la différence entre les valeurs moyennes de $\mathrm{P}$ pour les deux variétés 1-55 Single et 34-13 double est significative et ce avec un coefficient de sécurité de $96 \mathrm{p}$. Ioo.

2. Le même raisonnement se rapportant au paramètre $\mathrm{E}$ nous indique:

$t_{1,2}$ est inférieur à 2 .

$$
\begin{aligned}
& \mathrm{E}_{1}=23,230 \quad v_{1}=2,24 \quad n_{1}=100 \\
& \mathrm{E}_{2}=23,570 \quad v_{2}=2,44 \quad n_{2}=\mathrm{IOO} \\
& \mathrm{S} d=0,2 \mathrm{I} \\
& t_{1,2}=\mathrm{I}, 6
\end{aligned}
$$

Pour la grandeur E, la différence entre les valeurs moyennes des deux variétés st donc moins significative.

Le même raisonnement appliqué :

I. A la comparaison des variétés $\left\{\begin{array}{l}146 \\ \text { Double Fk Turc-Montpellier }\end{array}\right.$
2.
113 Single des Alpes
- 27 Single du Jura
3.
- - $\left\{\begin{array}{l}1-55 \text { Single } \\ 146\end{array}\right.$
4.
$-\quad\left\{\begin{array}{l}1-55 \text { Single } \\ 113 \text { Single }\end{array}\right.$
113 Single des Alpes
5.
- $\quad$ 1-55 Single
Double Fk Turc-Montpellier
6.
- $\left\{\begin{array}{l}34-13 \text { double } \\ 146\end{array}\right.$
7 .
- $\left\{\begin{array}{l}34-13 \text { double } \\ \text { Double Fk Turc-Montpellier }\end{array}\right.$

Annales de l'Abeille. - 1966. 
TABLEAU 5

\begin{tabular}{|c|c|c|c|c|}
\hline & \multirow{2}{*}{ Différences significatives } & \multirow{2}{*}{$\begin{array}{c}\text { Pour les } 2 \text { mesures } \\
\text { P et } \mathrm{E}\end{array}$} & \multicolumn{2}{|c|}{ Pour une seule mesure } \\
\hline & & & $\mathrm{P}$ & $\mathrm{E}$ \\
\hline$\Lambda$ & $\begin{array}{l}1-55 \text { Single } \\
34-13 \text { Double }\end{array}$ & - & + & 一 \\
\hline B & $\begin{array}{c}1 t 6 \\
\text { Double Fk-Turc - Mcnipellier }\end{array}$ & + & + & + \\
\hline $\mathrm{C}$ & $\begin{array}{l}113 \text { Single des Alpes } \\
27 \text { Single du Jura }\end{array}$ & + & + & + \\
\hline $\mathrm{D}$ & $\begin{array}{c}1-55 \text { Single } \\
146\end{array}$ & + & + & + \\
\hline $\mathrm{E}$ & $\begin{array}{l}\text { 1-5.5 Single } \\
113 \text { Single des Alpes }\end{array}$ & + & + & + \\
\hline $\mathrm{F}$ & Donble Fk $\frac{1-55 \text { Single }}{\text { Turc - Monipellier }}$ & + & + & + \\
\hline $\mathrm{G}$ & $\begin{array}{l}\text { 1-55 Single } \\
27 \text { Single du Jura }\end{array}$ & 一 & 一 & + \\
\hline $\mathrm{H}$ & $\begin{array}{l}34-13 \text { Double } \\
146\end{array}$ & + & + & + \\
\hline $\mathrm{I}$ & Double Fk $\begin{array}{c}\text { 34-13 Double } \\
\text { Turc - Montpellier }\end{array}$ & + & + & + \\
\hline $\mathrm{J}$ & $\begin{array}{l}34-13 \text { Double } \\
113 \text { Single des Alpes }\end{array}$ & + & +- & + \\
\hline $\mathrm{K}$ & $\begin{array}{l}34-13 \text { Double } \\
27 \text { Single du Jura }\end{array}$ & 一 & - & + \\
\hline $\mathrm{L}$ & $\begin{array}{c}146 \\
113 \text { Single des Alpes }\end{array}$ & + & + & + \\
\hline $\mathrm{M}$ & 27 Single du Jura & + & + & + \\
\hline $\mathrm{N}$ & $\begin{array}{c}\text { Double } F k-\text { Turc - Monlpellier } \\
113 \text { Single des Alpes }\end{array}$ & 一 & + & $\ldots$ \\
\hline 0 & $\begin{array}{c}\text { Double Fk- Turc - Montpellier } \\
27 \text { Single du Jura }\end{array}$ & + & + & + \\
\hline
\end{tabular}



8. A la comparaison des variétés $\left\{\begin{array}{l}34-13 \text { double } \\ 113 \text { Single des Alpes }\end{array}\right.$
9.
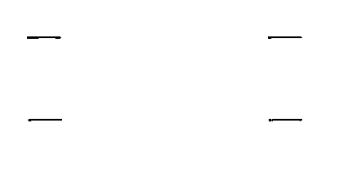
146
113 Single des Alpes
IO.
146
27 Single du Jura
II.
f Double Fh Turc-Montpellier
27 Single du Jura

montre que les différences entre les valeurs moyennes de $\mathrm{P}$ d'une part, de $\mathrm{E}$, d'autre part, concernant chaque groupe de deux variétés sont significatives, le coefficient de sécurité est toujours de 96 p. Ioo.

Par contre, la même étude portant sur la comparaison des variétés 1-55 Single et 27 Single $d u$ Jura d'une part, 34-13 double et $2^{7}$ Single du Jura d'autre part, montre que si la différence est significative en ce qui concerne le paramètre $\mathrm{E}$, elle ne l'est pas du tout si nous nous adressons au paramètre $P$, pour lequel $t_{1,2}$ est égal dans les deux cas à $I, 5$ et $I, 7$, donc inférieur à 2 .

Enfin, dernier cas, l'étude comparée des variétés double Fk Turc-Montpellier et 113 Single des Alpes d'une part, 1-55 Single et 34-13 double d'autre part montre que la différence est tout à fait significative lorsqu'on s'adresse au paramètre $\mathrm{P}$, par contre, ne l'est nullement si ce sont les valeurs de E que l'on compare : $t_{1,2}$, dans les deux cas, en effet, est égal à $\mathrm{I}, 2$ et $\mathrm{r}, 6$, donc inférieur à 2 .

\section{Conclusion.}

Si l'on excepte les 4 cas précédents pour lesquels l'étude comparée des deux paramètres $\mathrm{P}$ et $\mathrm{E}$ n'est discriminative que pour l'une des deux grandeurs $\mathrm{P}$ ou $\mathrm{E}$, nous constatons, au contraire, que la différence se montre significative à la fois pour $\mathrm{P}$ et $\mathrm{E}$ dans les onze autres cas de comparaison de variétés prises deux à deux. (tabl. 5)

Les différences entre deux valeurs moyennes de $P$ ou entre deux valeurs moyennes de $\mathrm{E}$, ne peuvent pas être attribuées uniquement à des fluctuations dues au hasard

\begin{tabular}{c|c}
\hline Variétés & $\begin{array}{c}\text { Grains de pollen avortés } \\
\text { sur } 500 \text { grains comptés }\end{array}$ \\
\hline 146 & 80 \\
\hline $1-55$ Single & 45 \\
\hline 113 Single des Alpes & 16 \\
\hline 27 Single du Jura & 16 \\
\hline Double-FK - Turc-Montpellier & 11 \\
\hline $34-13$ Double & 5 \\
\hline
\end{tabular}


et elles sont trop importantes pour que nous puissions admettre que les deux échan tillons proviennent d'une population unique.

$L$ 'évaluation des grandeurs $P$ et $E$ permet donc théoriquement dans tous les cas de différencier statistiquement telle variété de telle autre.

\section{Remarque.}

Nous nous sommes demandé s'il était possible de caractériser chaque variété d'Onobrychis sativa LAMK par le pourcentage de grains de pollen avortés. Ces grains apparaissent peu réfringents, fripés, ils semblent dépourvus de tout contenu vivant.

Or, chez certaines variétés le taux de pollens avortés semble particulièrement élevé.

Le pourcentage de pollens avortés (tabl. 6) semble assez faible, sauf en ce qui concerne les variétés 146 et $1-55$ Single. Ce critère ne semble pas suffisant pour caractériser chaque variété, il peut cependant constituer un caractère "secondaire" lorsqu'il s'agit de la détermination des deux premières variétés.

\section{B - Onobrychis sativa LAMK. ssp. Onobrychis supina DE CANDOLLE}

Préparations faites à Bures-sur-Yvette (I964), échantillon fourni par le Muséum d'Histoire naturelle, recueilli dans la région de Digne.

Symétrie et forme.

Pollen isopolaire, tricolpé, prolate, circulaire en vue polaire.

Dimensions.

\section{Apertures.}

$$
\begin{aligned}
& \overline{\mathrm{P}}=35,08 \pm 0,3 \mathrm{I} \mu \\
& \overline{\mathrm{E}}=20,85 \pm 0,07 \mu
\end{aligned}
$$

Trois sillons ; ces sillons portent en leur milieu une bande sombre presque continue d'ectexine. Les bords sont nets et réguliers, les marges sont peu épaisses.

Exine.

$$
\begin{aligned}
& \varepsilon=3,8 \pm 0,8 \mu \\
& t=4,4 \pm 0,8 \mu
\end{aligned}
$$

Réticulée, les " muri " du réseau sont épais, les lumina ont un diamètre $₹ I, 55 \mu$, les columelles sont bien visibles.

Intine.

Mince.

L'ensemble exine et intine a une épaisseur d'environ $\mathrm{I}, 55 \mu$. Cytoplasme.

Très granuleux et jaunâtre.

C - Onobrychis sativa LAMK. var. montana (GRENIER-GODRON)

Préparations faites à Bures-sur-Yvette (I964). F́chantillon fourni par le Muséum d'Histoire naturelle, provenant du Lautaret, Hautes-Alpes. 
Symétrie et forme.

Pollen isopolaire, prolate, tricolpé, circulaire en vue polaire.

Dimension.

$$
\begin{aligned}
& \overline{\mathrm{P}}=35,08 \pm 0,20 \mu \\
& \overline{\mathrm{E}}=2 \mathrm{I}, 0 \mathrm{I} \pm 0,00 \mu
\end{aligned}
$$

Apertures.

Trois colpus présentant des bords réguliers, recouverts en leur milieu par l'ectexine.

$$
\varepsilon=4,3 \pm 0,8 \mu
$$

Les sillons sont caractéristiques par leur grande largeur.

$$
t=4,3 \pm 0,8 \mu
$$

Exine.

Réticulée, le réseau est régulier, les columelles sont visibles.

Intine.

Très claire, a une épaisseur égale à celle de l'exine.

\section{Cytoplasme.}

Légèrement grantuletx.

$$
\text { D -- Onobrychis caput-galli (LAMARK) }
$$

Préparations faites à Bures-sur-Yvette (1964) - échantillon du Museum d'Histoire naturelle, provenant des environs de Lisbonne.

Symétrie et forme.

Pollen isopolaire, tricolpé, prolate, circulaire en vue polaire.

Dimensions.

$$
\begin{aligned}
& \overline{\mathrm{P}}=36, \mathrm{I} 9 \pm 0,28 \mu \\
& \overline{\mathrm{E}}=2 \mathrm{I}, \mathrm{OI} \pm 0, \mathrm{OI} \mu
\end{aligned}
$$

Apertures.

Trois colpus à bords nets et réguliers, les marges sont peu épaisses $(I, 5$ à 2,3$)$.

$$
\begin{aligned}
& \varepsilon=3, \mathrm{I} \pm 0,8 \mu \\
& t=4,4 \pm 0,8 \mu
\end{aligned}
$$

Exine.

Régulièrement réticulée, les columelles sont peu visibles, les lumina du réseau sont plus petites le long des sillons que dans les intercolpia. L'ectexine est sombre tandis que l'endexine est claire. 
Intine.

Mince et claire.

L'ensemble exine + intine \# $\mathrm{I}, 55 \mu$.

Cyloplasme.

Très finement granuleux.

\section{$\mathrm{E}$ - Onobrychis aequidentata (DUMONT D'URVILLE)}

Préparations faites à Bures-sur-Yvette. Échantillon du Muséum d'Histoire naturelle, en provenance des environs de Marseille.

Cette espèce est très rare.

Symétrie et forme.

Pollen isopolaire, tricolpé, prolate, circulaire en vue polaire.

Pollen à pôles plus effilés et à contour irrégulier.

Dimensions.

$$
\begin{aligned}
& \overline{\mathrm{P}}=42,90 \pm 0,44 \mu \\
& \overline{\mathrm{E}}=24, \mathrm{OI} \pm \mathrm{o}, \mathrm{OI} \mu
\end{aligned}
$$

Apertures.

Trois colpus nets et réguliers.

$$
\begin{aligned}
& \varepsilon=3, \mathrm{I} \pm 0,8 \mu \\
& l=4,6 \pm 0,8 \mu
\end{aligned}
$$

Exine.

Reticulum régulier, les mailles du réseau sont plus petites sur les bords des sillons que dans les intercolpia.

L'endexine est claire, l'ectexine est foncée.

Intine.

Mince et claire.

Cyloplasme.

Granuleux.

$$
\mathrm{F} \text { - Onobrychis saxatilis (L.AMARK) }
$$

Préparations faites à Bures-sur-Yvette (I964). Échantillon du Muséum d'Histoire naturelle, provenant des Hautes-Alpes (route de Briançon à Cervières).

Symétrie et forme.

Pollen isopolaire, tricolpé, prolate, circulaire en vue polaire.

Dimensions.

$$
\begin{aligned}
& \overline{\mathbf{P}}=32,50 \pm 0,20 \mu \\
& \overline{\mathrm{E}}=\mathrm{I} 8, \mathrm{OI} \pm \mathrm{O}, \mathrm{OI} \mu
\end{aligned}
$$


Apertures.

Trois sillons à bords nets et réguliers, chaque sillon porte en son milieu une bande étroite et sombre d'ectexine.

$$
\begin{aligned}
& \varepsilon=3, \mathrm{I} \pm 0,8 \mu \\
& t=4,4 \pm 0,8 \mu
\end{aligned}
$$

Exine.

Réticulée, les lumina du réseau sont très petites.

Les columelles sont visibles.

Intine.

Claire et mince.

L'ensemble exine-intine a une épaisseur $\leqslant I, 55 \mu$.

Cytoplasme.

Finement granuleux.

$$
\mathrm{G} \text { - Onobrychis crista-galli (LAMARK) }
$$

Préparations faites à Bures-sur-Yvette Échantillon fourni par le Muséum d'Histoire naturelle, provenant d'Italie, Sorrente et Castellamare.

Symétrie et forme.

Pollen isolopolaire, prolate, tricolpé, circulaire en vue polaire .

\section{Dimensions :}

$$
\begin{aligned}
& \overline{\mathrm{P}}=47, \mathrm{IO} \pm 0,59 \mu \\
& \overline{\mathrm{E}}=24, \mathrm{OI} \pm 0, \mathrm{OI} \mu
\end{aligned}
$$

Apertures.

Les trois sillons sont moins nettement définis que dans les cas précédents, la ligne sombre d'ectexine que porte chaque sillon en son milieu est à peine visible.

$$
\varepsilon=3, \mathrm{OI} \pm 0,8 \mu
$$

Les extrémités de deux sillons consécutifs sont rarement visibles en vue polaire, $t$ n'a pas été mesuré.

Exine.

L,es mailles du réticulum sont petites et les muri sont épais, aussi ce réticulum est-il très dense.

Les columelles sont visibles.

\section{Intine.}

Très mince et claire. 
Cyltoplasme.

Très granuleux.

Nous avons regroupé dans le tableau 7 l'ensemble de ces résultats.

TABLEAU 7

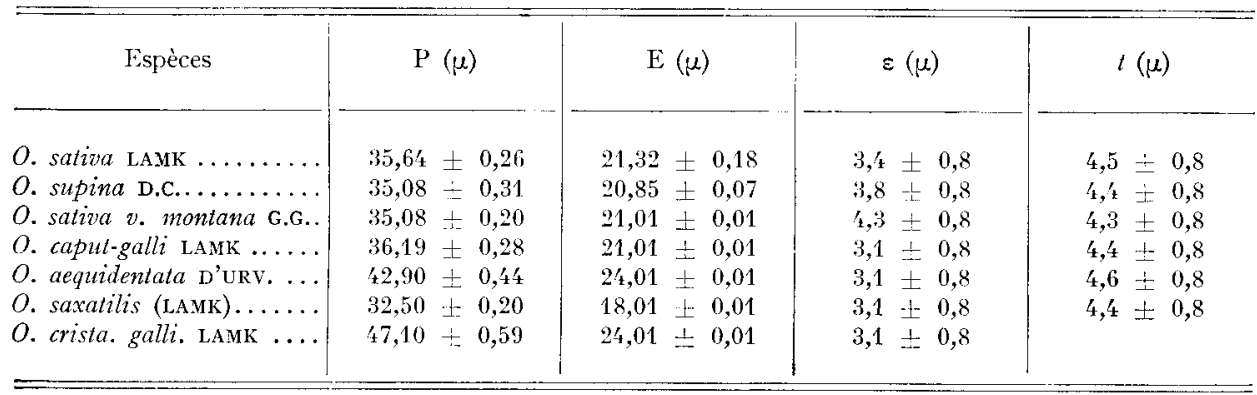

\section{II. - ÉTUDE DES GRAINS DE POLLEN D' " ONOBRYCHIS » RENCONTRÉS DANS I,ES MIELS DE, CERTAINES RÉGIONS DE France et D'Espagne}

L'étude morphologique des pollens des différentes espèces d'Onobrychis a été complétée par la recherche de ces pollens dans des préparations effectuées à partir d'échantillons de miel prélevés dans la collection du laboratoire - échantillons dont l'origine avait été antérieurement vérifiée.

Les échantillons choisis provenaient de régions différentes et avaient tous $O n o-$ brychis pour pollen dominant.

Nous avons essayé, par la même méthode statistique que celle utilisée pour caractériser les pollens et par une méthode graphique, de distinguer, dans les miels, les différentes espèces d'Onobrychis en rapprochant les pollens des types morphologiques précédemment décrits.

L'étude graphique consiste à représenter les espaces de variation entourant les valeurs moyennes de $P$ et de $E$, pour chaque espèce et variété d'Onobrychis connues, ainsi que les espaces de variation entourant les valeurs moyennes de $\mathrm{P}$ et de $\mathrm{E}$ pour chaque miel étudié. (fig. 9).

\section{I. Étude des miels de l'Indre-et-Loire (3 échantillons)}

Les grandeurs sur lesquelles repose la distinction des différents pollens d'Onobrychis sont principalement $\mathrm{P}$ et $\mathrm{E}$. Les mesures de ces deux paramètres sont effectuées sur Ioo grains par échantillon de miel : nous obtenons dans ces conditions les valeurs moyennes suivantes:

$$
\begin{aligned}
& \mathrm{P}=35,54 \pm 0,30 \mu \\
& \overline{\mathrm{E}}=2 \mathrm{I}, \mathrm{I} 2 \pm 0, \mathrm{IO} \mu
\end{aligned}
$$




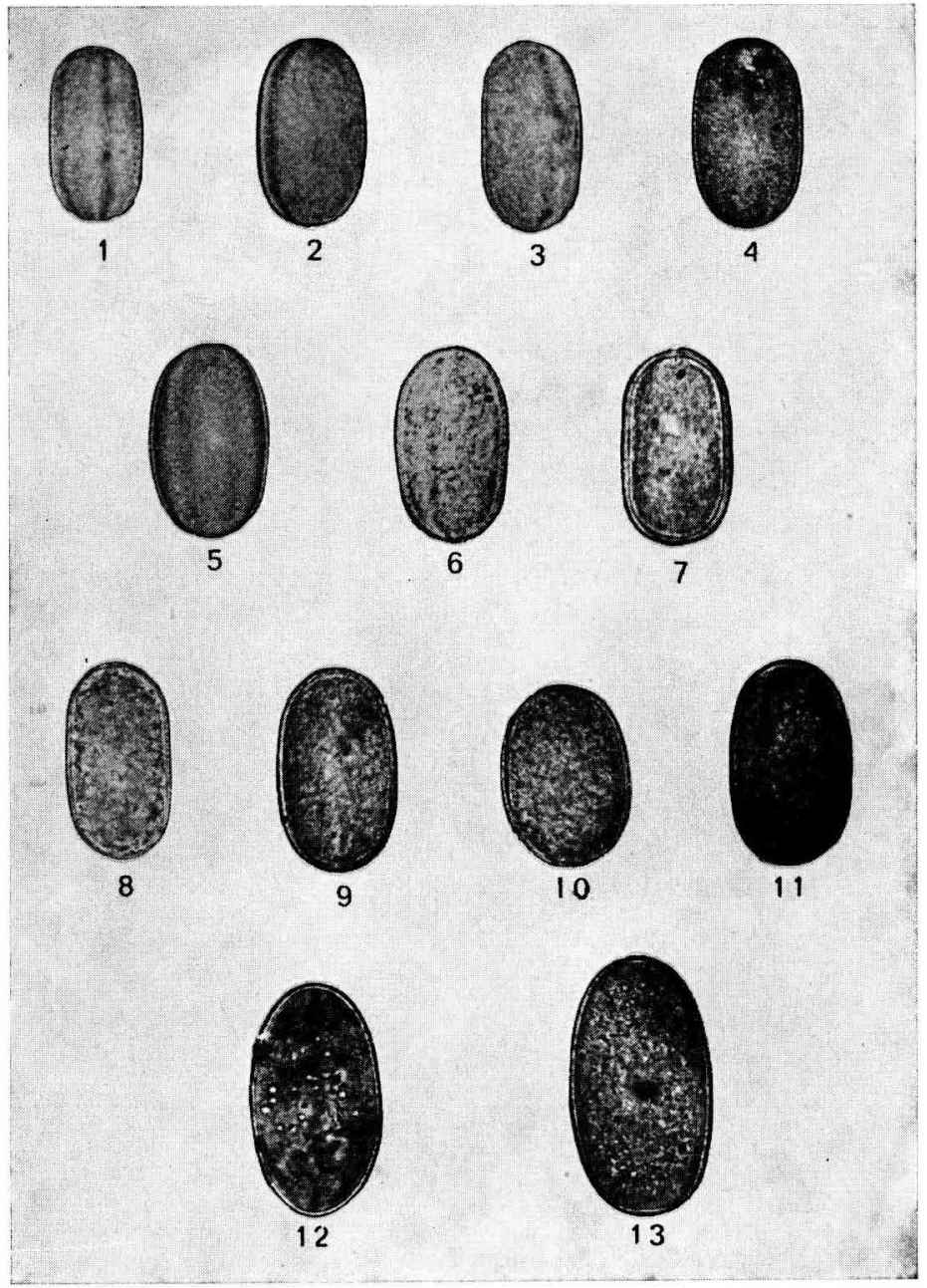

FIG. 8

I O. saxatilis LAMK

2 O. sativa v. 27 Single du Jura

3 O. sativa supina D. C.

4 O. sativa montana G. G.

5 O. caput-galli

6 O. sativa LAMK

7 O. sativa v. 113 Single des Alpes
8 O. sativa v. Double Fk Turc-Montpellier $9 O$. sativa v. 146

Io 0 . sativa v. 16-55 Single

I I $O$. sativa v. 34-13 Double

$12 O$. aquidentata

I3 O. crista-galli 


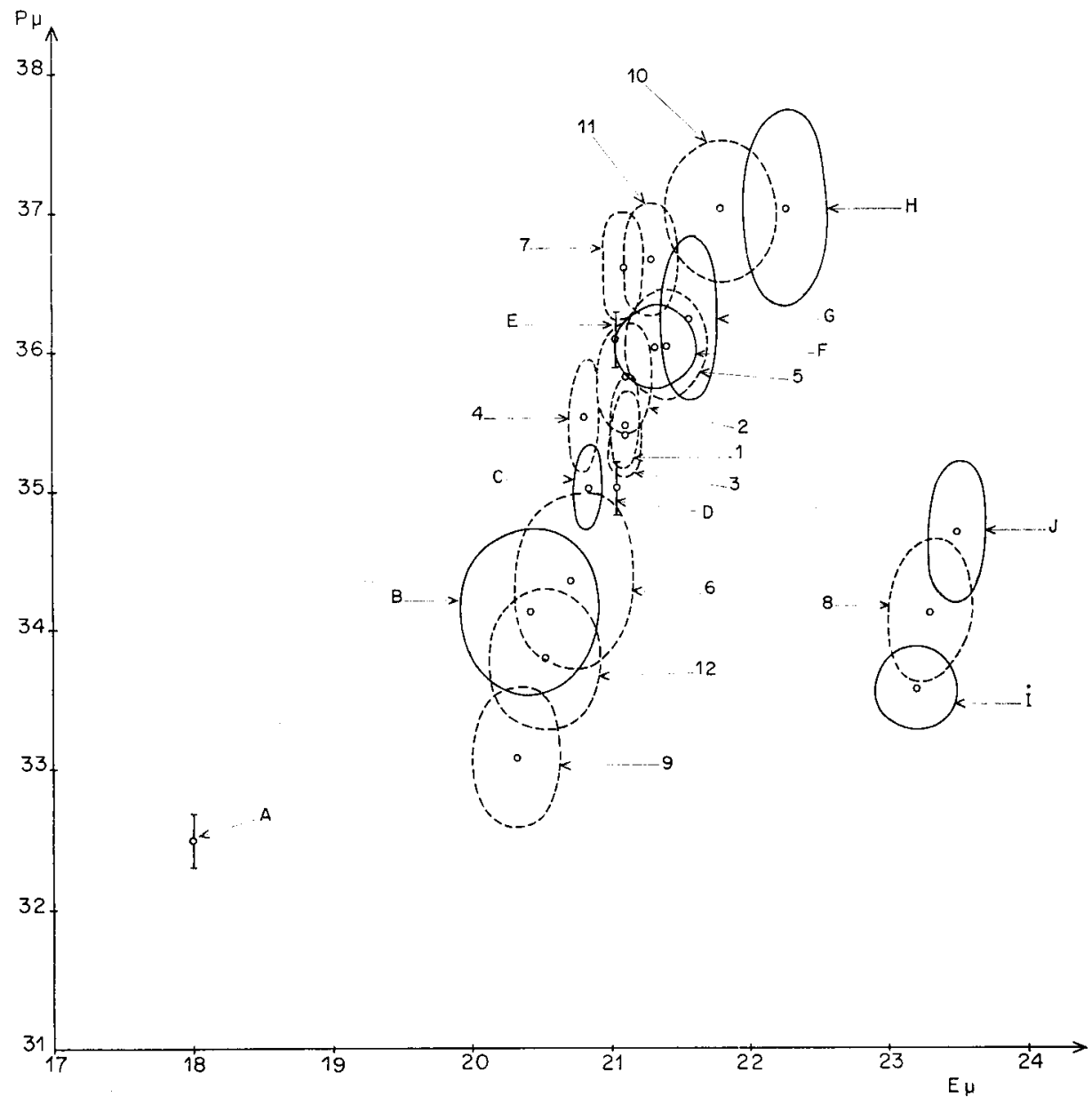

Fig. 9. - Etude des espaces de variation

Pollens rencontrés dans les miels provenant de (-......).
I. Indre-et-Loire.
7. Hérault.
2. Aisne.
8. Aveyron.
3. Touraine
9. Vaucluse.
4. Gâtinais.
Io. Var.
5. Basses-Alpes.
I I. Puy-de-Dôme.
6. Jura
I2. Espagne.
Variétés et espèces d'Onobrychis (-).
A. Onobrychis saxatilis.
B. Variété 27 Single du Jura.
F. Variété 113 Single des Alpes.
C. Onobrychis supina.
G. Variété Double Fk Turc-Monipellier
1. Variété Montana.
H. Variété 146.
E. Onobrychis caput-galli.
I. Variété 1-55 Single.
J. Variété 34-13 Double. 
Le relevé des diffétentes mesures faites sur roo grains montre que :

60 p. roo des grains ont une valeur de $\mathrm{P}$ proche de $35,005 \mu$

et

$98 \mathrm{p}$. Ioo des grains ont une valeur de $\mathrm{E}$ proche de $2 \mathrm{I}, 005 \mu$

La dispersion est donc faible. De plus, nous constatons que l'espace de variation concernant les pollens rencontrés dans ces miels recoupe celui des pollens d'Onobrychis sativa I,AMK, voire même celui d'une variété qui aurait les mêmes caractéristiques biométriques que la variété 113 Single des Alpes.

Ainsi, nous pouvons dire que les grains de pollen rencontrés dans les miels de l'Indre-et-L Lire présentent les mêmes caractères que ceux de l'espèce sativa ( $\mathrm{LAMK}$,) Or cette espèce est abondamment cultivée, sauf en Bretagne et se trouve à l'état spontané dans presque toute la France; il semble donc normal de rencontrer le pollen d'O. sativa LAMK dans ces miels.

De la même manière, nous analysons :

\section{Les miels de l'Aisne (2 échantillons)}

Les calculs statistiques nous donnent comme valeurs moyennes de $\mathrm{P}$ et de $\mathrm{E}$ :

$$
\begin{aligned}
& \overline{\mathrm{P}}=35,82 \pm 0,42 \mu \\
& \overline{\mathrm{E}}=2 \mathrm{I}, \mathrm{I} 8 \pm 0,24 \mu
\end{aligned}
$$

en accord avec le relevé des mestures qui nous indique que:

$50 \mathrm{p}$. Ioo des grains ont une valeur voisine de $35,005 \mu$ pour $\mathrm{P}$ et $\quad 80 \mathrm{p}$. Ioo des grains ont une valeur voisins de $2 \mathrm{I}, 005 \mu$ pour $\mathrm{E}$

Graphiquement, nous constatons que l'espace de variation de $\mathrm{P}$ et de $\mathrm{E}$ concernant ces grains est presque totalement superposé aux espaces de variation ayant trait à l'espèce $O$. sativa LAmk et à la variété 113 Single des Alpes. De même qu'en ce qui concerne la région précédente, les pollens rencontrés dans ces miels possèdent les mêmes caractères morphologiques que ceux d'O. sativa LAMK., de la variété 113 Single des Alpes, espèce et variété cultivées dans ces régions.

\section{Les miels de Touraine ( $\left.{ }^{1}\right)$ (2 échantillons)}

Les mesures réalisées ont donné les valeurs moyennes suivantes :

en précisant que

$$
\begin{aligned}
& \overline{\mathrm{P}}=35,46 \pm 0,34 \mu \\
& \overline{\mathrm{E}}=2 \mathrm{I}, \mathrm{I} 4 \pm 0, \mathrm{I} 4 \mu
\end{aligned}
$$

et

60 p. Ioo des grains ont $P \simeq 35,005 \mu$

98 p. Ioo des grains ont $\mathrm{E} \simeq 2 \mathrm{I}, 005 \mu$

L'espace de variation se confond avec celui ayant trait aux miels de l'Indreet-Loire. Les pollens ont les mêmes caractères biométriques dans les deux cas et ressemblent ainsi à ceux d'O. sativa LAMK et à ceux de la variété 113 Single des Alpes.

(1) C'est-à-dire l'Tndre-et-Loire et les départements limitrophes, en partie. 


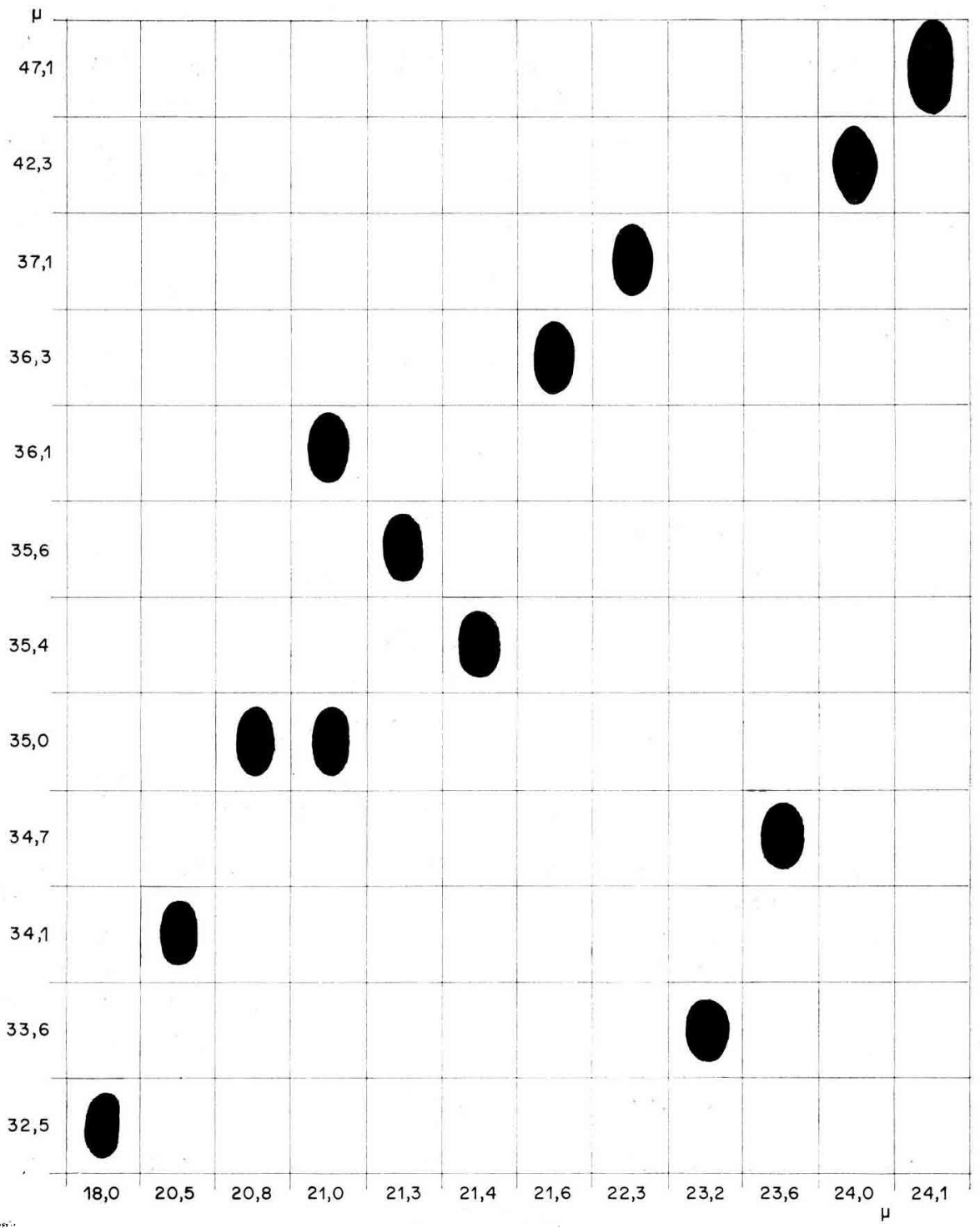

Fig. Io. - Répartition des différents types en fonction de $P$ et de $E$ : verticalement : $\mathrm{P}$

horizontalement : $\mathrm{E}$.

Pour E : 18,0 $\mu-O$. saxatilis $20,5 \mu-O$. sativa $20,8 \mu-0$. sativa $2 \mathrm{r}, \mathrm{o} \mu-$ en haut en bas $2 \mathbf{1}, 3 \mu-O$. sativa $21,4 \mu-O$, sativa $2 \mathrm{I}, 6 \mu-O$. sativa $22,3 \mu-O$. sativa $23,2 \mu$ - O. sativa $23,6 \mu-O$. sativa $24,0 \mu-O$. aequidentata $24, \mathrm{I} \mu-0$. crista-galli. var. 27 Single du Jura.

var. supina $D$. $S$.

O. sativa var. montana. O. caput galli

LAMK.

var. 311 Single des Alpes.

var. Double Fk Turc-Montpellier.

var. 146 .

var. 155 Single.

var. 34-13 Double. 
Comme nous pouvions le prévoir, nous ne rencontrons dans ces trois régions : Indre-et-Loire, Aisne, Touraine que l'espèce $O$. sativa LAMK., les pollens ont le même aspect que ceux de la variété très cultivée 113 Single des Alpes.

\section{Les miels du Gâtinais (2 échantillons)}

Le pollen de Sainfoin est retrouvé en abondance dans les miels du Gâtinais avec pour valeurs moyennes :

$$
\begin{aligned}
& \overline{\mathrm{P}}=35,58 \pm 0,47 \mu \\
& \overline{\mathrm{E}}=20,86 \pm 0, \mathrm{I} 3 \mu
\end{aligned}
$$

95 p. I00 des grains ont une valeur $34,005<\mathrm{P}<36,005 \mu$ et $\quad 98$ p. Ioo des grains ont une valeur $\quad E \simeq 2 \mathrm{I}, 005 \mu$

D'après l'étude graphique et les mesures effectuées, nous constatons que ces pollens 'ont les mêmes caractères biométriques que ceux d'O. sativa LAMK, et plus précisément ceux de la sous-espèce supina $\mathrm{D}$. C.

\section{Les miels des Basses-Alpes (5 échantillons)}

Les miels de cette région sont également très riches en pollen d'Onobrychis.

Les valeurs moyennes de $\mathrm{P}$ et de $\mathrm{E}$ sont :

$$
\begin{aligned}
& \overline{\mathrm{P}}=36, \mathrm{oI} \pm 0,45 \mu \\
& \overline{\mathrm{E}}=2 \mathrm{I}, 24 \pm 0,36 \mu
\end{aligned}
$$

et

50 p. Ioo des grains ont une valeur de $\mathrm{P} \simeq 36,005 \mu$

40 p. roo des grains ont une valeur de $\mathrm{P} \simeq 38,005 \mu$

$90 \mathrm{p}$. roo des grains ont une valeur de $\mathrm{F} \simeq 2 \mathrm{I}, 005 \mu$

L'étude géographique nous amène à constater qu'il y a recoupement entre leur espace de variation et ceux concernant les espèces connues : O. sativa LAMk. et o. caput-galli LAMK.

Les miels renferment donc des pollens ayant les mêmes caractéristiques que ceux de :

O. sativa LAMk. variétés $\left\{\begin{array}{l}113 \text { Single des Alpes } \\ \text { Double Fk-Turc-Montpellier }\end{array}\right.$

et

O. caput-galli. LAMK

La rencontre, dans les miels de cette région, du pollen d'Onobrychis, caputgalli LAMK est-elle normale?

Si nous nous référons à l'aire de distribution géographique, nous constatons, en effet, qu'O. caput-galli se rencontre dans les lieux secs et arides de la Provence, du Languedoc et du Roussillon. Aussi, est-il normal de rencontrer des grains de pollen ayant les mêmes caractères que ceux d'O. caput-galli dans les miels récoltés dans les Basses-Alpes. 


\section{Les miels du Jura (2 échantillons)}

Les résultats obtenus pour les valeurs de $\mathrm{P}$ et de $\mathrm{E}$ sont :

$$
\begin{aligned}
& \overline{\mathrm{P}}=34,32 \pm 0,58 \mu \\
& \overline{\mathrm{E}}=20,74 \pm 0,38 \mu
\end{aligned}
$$

et

50 p. I00 des grains ont $34,005<\mathrm{P}<36,005 \mu$

$50 \mathrm{p}$. I00 des grains ont $20,005<\mathrm{E}<22,005 \mu$

Ces données et celles résultant de l'étude graphique nous indiquent que les miels de cette région renferment surtout des pollens apparentés à ceux de la variété 27 Single du Jura d'une part, et à la variété montana G.G. d'autre part.

La variété $O$. montana se rencontre, en effet, dans le sud du Jura. C'est une plante de prairies et de rocailles des hautes montagnes.

\section{Les miels de l'Hérault (3 échantillons)}

L,es mesures effectuées nous donnent les résultats suivants :

$$
\begin{aligned}
& \overline{\mathrm{P}}=36,96 \pm 0,40 \mu \\
& \overline{\mathrm{E}}=2 \mathrm{I}, \mathrm{I} 6 \pm 0, \mathrm{I} 4 \mu
\end{aligned}
$$

80 p. Ioo des grains ont une valeur $\mathrm{P} \simeq 36,005 \mu$ 90 p. Ioo des grains ont une valeur $\mathrm{E} \simeq 21,005 \mu$

Les résultats statistiques nous amènent à conclure que de nombreux grains de pollen présentent les caractères d'O. caput-galli LAMK., d'autres étant très voisins d'O. supina D.C.

Ces deux espèces se rencontrent dans les lieux arides du Midi, Provence, Languedoc, Roussillon. Il semble normal qu'elles puissent être abondamment visitées par l'Abeille.

\section{Les miels de l'Aveyron (3 échantillons)}

Les valeurs moyennes de $P$ et $E$, calculées comme précédemment, en ce qui concerne les miels de cette région, sont les suivantes:

$$
\begin{aligned}
& \overline{\mathrm{P}}=34, \mathrm{I} 2 \pm 0,59 \mu \\
& \overline{\mathrm{E}}=23,36 \pm 0,38 \mu
\end{aligned}
$$

et $\quad 50 \mathrm{p}$. Ioo des grains de pollen ont une valeur $\mathrm{P} \simeq 32,005 \mu$

$40 \mathrm{p}$. I00 des grains de pollen ont une valeur $\mathrm{P} \simeq 36,005 \mu$

80 p. I00 des grains de pollen ont une valeur $\mathrm{E} \simeq 22,005 \mu$

Les études statistiques et graphiques montrent que nous avons affaire essentiellement, dans ce cas, à des pollens voisins de ceux des variétés d'O. sativa LAMK. : 34-13 Double et 1-55 Single.

Les pollens de ces deux variétés sont très différents de ceux des autres variétés ; ils sont caractérisés par tune grande largeur et une petite longueur, raison pour laquelle ils ont été classés sous la dénomination "variétés courtes ", s'opposant par 
là aux autres variétés dites "longues" .Nous constatons que ces pollens ne se rencontrent que dans les miels de l'Aveyron; la culture de ces deux variétés d' $O$. sativa LAMK semble être localisée essentiellement dans cette région.

\section{Les miels du Vaucluse (4 échantillons)}

L'étude statistique nous fournit pour valeurs moyennes de $\mathrm{P}$ et de $\mathrm{E}$ :

$$
\begin{aligned}
& \overline{\mathrm{P}}=32,82 \pm 0,42 \mu \\
& \overline{\mathrm{E}}=20,36 \pm 0,38 \mu
\end{aligned}
$$

Parmi ces pollens, 50 p. Ioo présentent les caractères d'O. saxatilis LAMK $\mathrm{P} . \simeq 32,005 \mu$ et $\mathrm{E} \simeq \mathrm{I} 8,005 \mu$ et 50 p. Ioo ceux d'O. sativa $\mathrm{L}_{\mathrm{A}} \mathrm{Amk}$. Ainsi, il semble que les miels du Vaucluse renferment des pollens de 2 espèces d'Onobrychis :

Onobrychis sativa I.AMK. et Onobrychis saxatilis LAMK. O. sativa LAMK est cultivé et se trouve à l'état spontané dans presque toute la France et $O$. saxatilis LAMK se rencontre sur les côteaux rocailleux du Sud-Est, du Midi, dans le Dauphiné, la Provence, 1'Aude, les Pyrénées-Orientales. Rien de surprenant donc à ce que le pollen d'O. saxatilis LAMK soit rencontré dans les miels du Vaucluse.

\section{Io. Les miels du Var (5 échantillons)}

Nous remarquons que les pollens de Sainfoin retrouvés dans ces miels présentent la plus grande valeur moyenne de P, c'est-à-dire la plus grande longueur.

$$
\begin{aligned}
& \overline{\mathrm{P}}=37,03 \pm 0,50 \mu \\
& \overrightarrow{\mathrm{E}}=2 \mathrm{I}, 80 \pm 0,40 \mu
\end{aligned}
$$

80 p. Ioo des grains ont une valeur $36,005<\mathrm{P}<39,005 \mu$ et $\quad 80 \mathrm{p}$. I00 des grains ont une valeur $\mathrm{E} \simeq 2 \mathrm{I}, 005 \mu$

L'étude graphique confirme les résultats statistiques, ces grains de pollen présentent les mêmes caractères que ceux de la variété 146 d'O. sativa LAMK.

\section{Les miels du Puy-de-Dôme}

Les grains de pollen sont caractérisés par les valeurs moyennes :

$$
\begin{aligned}
& \overline{\mathrm{P}}=36,62 \pm 0,45 \mu \\
& \overline{\mathrm{E}}=2 \mathrm{I}, 32 \pm 0,20 \mu
\end{aligned}
$$

et 50 p. Ioo des grains ont une valeur 34,005 $<\mathrm{P}<36,005 \mu$

50 p. Ioo des grains ont tune valeur 37,005 $<\mathrm{P}<39,005 \mu$

80 p. I00 des grains ont une valeur $\quad \mathrm{E} \simeq 2 \mathrm{I}, 005 \mu$

L'étude graphique montre que l'espace de variation recoupe ceux des variétés Double Fk-Turc-Montpellier et 113 Single des Alpes.

Dans ces miels, les pollens sont donc à rapprocher de ceux d'Onobrychis sativa LAMK. 
I2. Les miels d'Espagne (4 échantillons)

L'évaluation des valeurs moyennes de $\mathrm{P}$ et de $\mathrm{E}$ nous indique :

$$
\begin{aligned}
& \overline{\mathrm{P}}=33,82 \pm 0,54 \mu \\
& \overline{\mathrm{E}}=20,52 \pm 0,42 \mu
\end{aligned}
$$

$\left\{\begin{array}{l}40 \mathrm{p} \text {. I00 des grains ont } 30,005<\mathrm{P}<33,005 \mu \\ 50 \text { p. I00 des grains ont } 34,005<\mathrm{P}<36,005 \mu \\ 50 \text { p. I00 des grains ont } \mathrm{E}_{1} \simeq 18,005 \mu \\ 50 \text { p. I00 des grains ont } \mathrm{E} \simeq 2 \mathrm{I}, 005 \mu\end{array}\right.$

Ces résultats et ceux de l'interprétation graphique nous permettent de dire que ces pollens présentent les mêmes caractères que ceux de la variété 27 Single du Jura de l'espèce $O$. sativa LAMk. Par ailleuts, nous rencontrons des petits grains comparables à ceux d'O. saxatilis L.A.MK.

De façon générale, dans les miels d'Espagne, les pollens de Sainfoin n'appa. raissent qu'à 1'état " isolé " ou " d'accompagnement ".

\begin{tabular}{|c|c|}
\hline Régions & Pollens d'Onobrychis L. comparables à ceux des espèces : \\
\hline 1 Indre-et-Loire. . & saitiva. LAMARK \\
\hline 2 Aisne $\ldots \ldots \ldots$ & saliva LAMK. var. 113 Single des Alpes \\
\hline 3 Touraine $\ldots$. & sativa LAMK. var. 113 Single des Alpes \\
\hline 4 Gâtinais & suiva LAMK. sous-espèce supina D.C. \\
\hline 5 Basses-Alpes .. & sativa LAMK. et caput-galli LAMK. \\
\hline 6 Jura $\ldots \ldots \ldots$ & sativa LaMK. var. 27 Single du Jura, var. montana \\
\hline 7 Hérault $\ldots .$. & saliva LAMK. et capul-galli LAMK. \\
\hline 8 Aveyron ..... & sativa LAмк. var. 1-55 Single et 34-13 Double \\
\hline 9 Vaucluse.... & sativa LAMK. et saxatilis LAMK. \\
\hline 10 Var $\ldots$. & sativa LAMK. var. 146 \\
\hline 11 Puy-de-Dôme... & sativa LAMK. var. Double $F k-$ Turc -- Montpellier \\
\hline 12 Espagne & sativa LAMK. et saxalilis LAMK. \\
\hline
\end{tabular}

TABLEAU 8

1 Conclusion. (tab1. 8)

I - O. sativa LAMK. est rencontré dans tous les échantillons examinés, sa présence constante s'accorde bien avec sa répartition géographique : l'espèce apparaissant cultivée ou spontanée sur l'ensemble du territoire français. 
I. Dans certaines régions : Indre-et-Loire, Touraine, Gâtinais, Aisne, Jura, Aveyron, Var, Puy-de-Dôme, l'espèce $O$. sativa LAMk paraît être le seul Sainfoin présent dans les miels.

2. Dans les Basses-Alpes et l'Hérault, cette espèce semble être accompagnée d'O. caput-galli LAMK dont l'aire de répartition géographique est très localisée (Littoral méditerranéen, Provence, Languedoc, Roussillon).

3. Dans le Vaucluse et en Espagne enfin, nous trouvons l'association $O$. saxatilis LAMK-O. sativa LAMK.

II - Cette étude nous montre que deux espèces d'Onobrychis semblent ne pas intéresser 1'Abeille ou très peu :

O. aequidentata D'URV. et $O$. crista-galli LAMK.

O. aequidentata D'URV. est très rare, localisée dans le vallon de Roubaou près de Marseille.

O. crista-galli LAMK., sous-espèce de caput-galli LAMK., se rencontre moins souvent que ce dernier dans les mêmes régions du littoral méditerranéen. I1 s'agit là encore d'une plante peu commune.

\section{DISCUSSION}

I. - Les résultats apportés par l'étude statistique des grandeurs $\mathrm{P}$ et $\mathrm{E}$ se rapportant aux pollens des différentes variétés cultivées d'Onobrychis sativa LAMK nous montrent que :

I. Les 6 variétés étudiées se classent en deux groupes; d'une part les variétés dites "longues";

$$
\text { dont le rapport } \frac{\mathrm{P}}{\mathrm{E}} \# \mathrm{I}, 65\left\{\begin{array}{l}
146 \\
113 \text { Single des Alpes } \\
27 \text { Single du Jura } \\
\text { Double F K-Turc-Montpellier }
\end{array}\right.
$$

d'autre part, les variétés dites "courtes",

$$
\text { dont le rapport } \frac{\mathrm{P}}{\mathrm{E}} \# \mathrm{I}, 50\left\{\begin{array}{l}
1-55 \text { Single } \\
34-13 \text { double }
\end{array}\right.
$$

2. Il n'existe aucune corrélation entre les variations de $P$ et de $E$ pour chaque variété saut pour denx d'entre elles: $34-13$ double et 27 Single du Jura où aux plus grandes valeurs de $\mathrm{P}$ correspondent les plus grandes valeurs de $\mathrm{E}$.

3. Les différences entre deux valeurs moyennes de $P$ ou entre deux valeurs moyennes de $\mathrm{E}$, ne sont pas dues au hasard, les 2 échantillons considérés ne proviennent pas d'une population unique.

La différence trouvée entre les moyennes de $\mathrm{P}$ et de $\mathrm{E}$ concernant deux variétés est dono significative.

Annales de l'Abeille. - 1966. 
Une distinction entre variétés reposant seulement sur l'évaluation des grandeurs de $P$ et de $E$ s'avère ainsi théoriquement possible.

II - La description des pollens des diférentes espèces d'Onobrychis permet de constater que tous présentent les mêmes caractères morphologiques concernant:

I. Le sporoderme : structure et sculpture de l'exine.

2. Les apertures $\left\{\begin{array}{l}\text { forme du sillon; } \\ \text { présence d'ectexine à l'intérieur du sillon ; } \\ \text { distance séparantles extrémités de } 2 \text { sillons consécutifs (soit } t \text { ) ; } \\ \text { largeur du sillon à l'équateur (soit } \varepsilon \text { ). }\end{array}\right.$

la seule exception à mentionner se rapporte à la variété Onobrychis montana G.G. Cette variété, en effet, se différencie notamment par la largeur de ses sillons :

$$
\varepsilon=4,4 \mu \text { ailleurs } \varepsilon=3, \mathrm{I} \mu \text {. }
$$

Les mesures de $P$ et $E$ représentent donc les deux seuls critères permettant de différencier deux espèces:
O. supina D. C.
$\mathrm{P} \# 35 \mu$
$\mathrm{E} \# 20 \mu$.
O. sativa $\mathrm{LAMK}$
$\mathrm{P} \# 35 \mu$
$\mathrm{E} \# 2 \mathrm{I} \mu$.
O. caput-galli LAMK
$\mathrm{P} \# 3^{6} \mu$
$\mathrm{E} \# 2 \mathrm{I} \mu$.
O. crista-galli LAMK
$\mathrm{P} \# 47 \mu$
$\mathrm{E} \# 24 \mu$.
O. aequidentata D'URV.
$\mathrm{P} \# 42 \mu$
$\mathrm{E} \# 24 \mu$.
$O$. saxatilis LAMKK
$\mathrm{P} \# 32 \mu$
$\mathrm{E} \# \mathrm{I} 8 \mu$.

Notons que les pollens d'Onobrychis crista-galli LAMK et d'Onobrychis aequidentata D'URV. apparaissent plus nettement différenciables des autres pollens d'Onobrychis, tant par leurs dimensions nettement plus élevées que par leur forme. Ces pollens sont, en effet, plus effilés aux deux extrémités, plus ventrus dans leur région équatoriale.

Ainsi, les pollens d'Onobrychis sativa LAMK, supina D. C., caput-galli LAMK, saxatilis LAMK ont schématiquement la forme d'un cylindre surmonté de deux demi-sphères, ceux de $O$. crista-galli I,AMK., $O$. aequidentata d'URV. sont plus ovoïdes.
O. sativa LAMK
O. supina D. C.
O. caput-galli LAMK
O. saxatilis LAMK

O. crista-galli LAMK

O. aequidentata D'URV.

III - Il se trouve que les pollens des deux espèces précisément plus différenciables ne se retrouvent pas à 1'analyse mélissologique et donc seule une étude biométrique permet de distinguer dans les miels les pollens des espèces et variétés d'Onobrychis communément rencontrés.

IV - Phylogénie. Un certain nombre de genres de Papilionacées: Coronilla, Hypocrepis, Ornithopus, Scorpioides, Hedysarun, Onobrychis, possèdent des feuilles à folioles disposées par paires avec une foliole terminale, caractère qui leur a valu d'être rapprochés les uns des autres. Or, si les cinq premiers genres précités ont un fruit qui se divise transversalement en articles qui se détachent chacun sans s'ouvrir 
avec la graine qu'il renferme, au contraire, chez Onobrychis, le fruit est réduit à un seul article ne s'ouvrant pas. Mais ce genre, par tous ses autres caractères, est relié à Hedysarum.

Il est intéressant de constater qu'à ces liens morphologiques viennent s'adjoindre des liens de type palynologique. Le pollen d'Hedysarum est, en effet, parmi les Papilionacées, celui qui se rapproche le plus du pollen d'Onobrychis ; il s'agit, en effet, de pollen isopolaire, nettement prolate, subcirculaire en vue polaire, l'ornementation de l'exine ne différant pas de celle d'Onobrychis si ce n'est par la taille des mailles du. réticulum ici plus petites. Fondamentalement, les deux genres diffèrent par la taille de leurs grains (plus petit chez Hedysarum) et aussi par la présence, au moins chez Hedysarum coronarium L. de trois pores superposés aux trois sillons : le pollen est tricolporé. Le pollen d'Hedysarum obscurum $\mathrm{L}_{\text {, }}$. ne possède, cependant, en guise d'apertures, que trois sillons : il est tricolpé tout comme celui d'Onobrychis.

Il semble donc que, partant du type Onobrychis tricolpé pour arriver au type des Papilionacées en général tricolporé, le pollen d'Hedysarum puisse être considéré comme un terme de passage : Hedysarum obscurum L. étant resté tricolpé, Hedy-

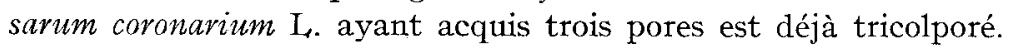

\section{Reçu pour publication en juin 1966.}

\section{REMERCIE,MENTS}

Je remercie très sincèrement M. le pr EichHoRs d'avoir accepté de diriger mon travail et de présider à la soutenance de ce diplôme.

Mes remerciements vont aussi à M. le Pr VAzART qui a bien voulu me fournir le second sujet et faire partie du Jury.

Enfin, que M. Aymonin, du Muséum National d'Histoire naturelle, qui m'a permis d'obtenir un certain nombre d'échantillons nécessaires à la réalisation de la présente étude, trouve l'expression de mes très sincères remerciements.

\section{SUMMARY}

BIOMETRIC STUDY OF POLLEN OF FRENCH SPECIES OF TIIE GENUS « ONOBRYCHIS 》

The following measurements were made on the pollen grains :

$\mathrm{P}=$ length of the polar axis ;

$\mathrm{E}=$ diameter of the grain at the equator ;

$\varepsilon=$ width of the germinal furrow at the equator ;

$t=$ distance separating the extremities of the two consecutive furrows.

The mean value of $P$, or $\bar{p}$, has been established for each species by measuring too grains, and the range of variations has been calculated for these parameters. In general, there seems to be no correlation between the variations of $\mathrm{P}$ and $\mathrm{E}$.

The six sativa varieties studied are divided into "long " varieties, with a ratio of $\frac{\mathrm{P}}{\mathrm{E}} \# \mathbf{1} .65$ and " short "varieties, with a ratio of $\frac{\mathrm{P}}{\mathrm{E}}$ \# I.50. A distinction between varieties resting solely on the evaluation of the size of $\mathrm{P}$ and $\mathrm{E}$ is theoretically possible. The description of the pollens of the different species of Onobrychis allows it to be shown that all have the same morphological charac- 
teristics with regard to the sporoderm and the apertures. The pollens of Onobrychis crista-galli LAMK and of Onobrychis aequidentata D'URV, seem quite clearly differentiable from the other Onobrychis pollens, as much by their greater dimensions as by their form. Among the Papilionaceae the pollen of Hydesarum is that which is closest to the Onobrychis pollen.

\section{RÉFÉRENCES BIBLIOGRAPHIQUES}

Bonsier Cr., 1934. Filore complele illustrée en couleurs de France, Suisse, Belgique. Toine 3, 85-86, E. Orlhac, Paris.

Coste H., 1937. Flore descripline el illustrée de France. 'lome I, 412-414. Librairie des Sciences et des Arts. l'aris.

ERdtmand G., 1954. An introduclion to pollen analysis. Chap. 5, 43-54, Almqvist et Wiksell, Stockholm. Fournier P'., I946. Les quatre fores de France, 578-579. P. Lechevallior, Paris.

KLATZMani J., I955. La localisalion des cultures el des productions animales en firance, 369-370. Imprimerie nationale, Paris.

Maurizio A., Louveaux J., ig63. Méthodes d'analyse pollinique des miels. Ann. Abeille, 6, $75^{-76}$.

Willom M. et Lange J., is7o. Prodromus forae hispanicae, seu sinopsis methodica omnium planta. rum in Hispania sponte rascentium ael frequentius cultarum quae innotherunt. Vol. III, 253-268. Edit Schweizerbart, Stuttgart. 